\title{
Nigerian cultural beliefs about mental health conditions and traditional healing: a qualitative study
}

\section{Dung Ezekiel Jidong, Di Bailey, Tholene Sodi, Linda Gibson, Natéwindé Sawadogo, Deborah Ikhile, David Musoke, Munyaradzi Madhombiro and Marcellus Mbah}

\begin{abstract}
Purpose - This study aims to explore how cultural beliefs and traditions are integral to understanding indigenous mental health conditions (MHCs) and traditional healing (TH). However, Nigerian cultural beliefs about MHCs and TH are under-researched.

Design/methodology/approach - This study adopted a qualitative design using critical realist and social constructionist perspectives to explore Nigerian mental health-care practitioners (MHCPS) and lay participants' (LPS) views regarding MHCs and TH. Purposive and snowball sampling techniques were used to select 53 participants (MHCPS $=26 ;$ : $P S=27$; male $=32$; female $=21$ ) in four Nigerian cities (Ado-Ekiti, Enugu, Jos and Zaria). Data were collected using semi-structured interviews and analysed through thematic analyses.

Findings - The data sets revealed three overarching themes, namely, existing cultural beliefs about MHCs as spiritual curse; description of TH as the first treatment modality for MHCs; and perceived stigma associated with MHCs and help-seeking behaviours.

Originality/value - A study on Nigerian cultural beliefs and TH contributes meaningfully to mental health systems. Future research and policy initiatives could explore ways of optimising TH practices and community awareness programmes to increase access to mental health care in Nigeria.
\end{abstract}

Keywords Cultural beliefs, Mental health conditions, Practices, Traditional healing, Nigeria

Paper type Research paper

\section{Background}

Mental health conditions (MHCs) such as anxiety, depression or psychosis are increasingly becoming the leading cause of disease burden and disability globally (Weye et al., 2020). The World Health Organization (WHO) special initiative for universal mental health coverage suggests that MHCs cause early lived mortality of 10-20 years (WHO, 2019a, 2019b). For instance, there are global records of over 800,000 deaths annually due to suicide mortality alone with economic losses of over US\$1tn per year, with a disproportionate impact in lowand middle-income countries (WHO, 2019a, 2019b).

In Africa, MHCs are exacerbated due to widespread misconceptions, a low policy priority for mental health, inadequate human resources and facilities for mental health provision, human rights abuses and stigmatisation (Oshodi et al., 2014), the already weakened mental health services on the continent (Sankoh et al., 2018). It is projected that by 2050, West African countries would experience a 129\% increase in MHCs (Charlson et al., 2014). In the quest to reduce the increasing mental health disease burden in the region, Gureje et al. (2019) explored the potentials of partnership for mental health development in Sub-Saharan Africa (PaM-D). They proposed that the research component of PaM-D should focus on
(Information about the authors can be found at the end of this article.)
Received 19 August 2020 Revised 30 January 2021 Accepted 22 March 2021

The study acknowledges the contribution of $\mathrm{PhD}$ research supervisors (i) Professor Rachel Tribe (ii) Professor Aneta Tunariu and (iii) Dr Poul Rohleder. 2015 University of East London Excellence PhD Scholarship. 
collaborative shared care treatment between traditional and faith healers, in conjunction with the biomedical providers, to treat MHCs; whereas mental health has different cultural contexts in Africa, the focus of this paper is Nigeria.

Nigeria has one of the most deprived mental health-care systems globally (Abdulmalik et al., 2016; Gureje et al., 2015; Jidong and Sanger, 2018). With a population of over 209 million (Worldometers, 2020), it is estimated that 20\%-30\% of the population suffers from MHCs (Onyemelukwe, 2016; Suleiman, 2016). However, there are minimal human resources and facilities for mental health-care provision in Nigeria (Anyebe et al., 2019). Only one out of every five people with an $\mathrm{MHC}$ can access any care (Abdulmalik et al., 2019). Gureje et al (2006) report that over a 12-month period, only $10 \%$ of those with MHCs had received any form of treatment in Nigeria. Similar to other low- or middle-income countries, the Nigerian Government health budget allocates less than $2 \%$ to the prevention and treatment of MHCs (World Health Organization [WHO], 2019b). Furthermore, only 0.7\% of those who did not have a symptom profile recognised under the Diagnostic and Statistical Manual of Mental Disorders, Fourth Edition (DSM-IV) criteria but might have reported $\mathrm{MHCs}$ were in treatment. Within this latter population, a significant $0.4 \%$ received treatment from traditional healing $(\mathrm{TH})$. The use of $\mathrm{TH}$ by service users might be underestimated by Western biomedically trained practitioners such as medical doctors, psychiatrists or practitioners, psychiatrists or clinical psychologists. However, a recent study in 2020 revealed that $81.6 \%$ of female service users in Ibadan, Nigeria, use TH for its cultural compatibility, affordability and ease of access (Li et al., 2020). Service users are deemed to be people who seek help or treatment for MHCs.

The Nigerian TH originates from a long history of practices based on cultural beliefs firmly rooted in the indigenous knowledge (Igberase and Okogbenin, 2017; Takim et al., 2013; Sarfo, 2015). Knowledge acquired from TH is used in the identification and healing process of MHCs (WHO, 2013). TH practice may involve the use of herbs, farm produce or verbal incantations and sacrificial activities with animals to invoke the spirit that heals MHCs (Borokini and Lawal, 2014). The WHO defined mental health as the state of well-being in which individuals can cope with daily life stressors, realises their potentials to work productively and contribute fruitfully to their community (WHO, 2001). A comprehensive definition of $\mathrm{MHC}$ is nearly impossible from a universal perspective because culture-specific rules determine and define what an MHC is and what is not. However, this paper understands that a MHC is a psychological "dysfunction that a particular culture defines as inappropriate" (Horwitz, 2020, p.12).

Epidemiological research has shown that TH's availability in the general population in Africa is 1:500 compared with 1:40,000 medical doctors (WHO, 2013). This is because $\mathrm{TH}$ tends to be readily available, affordable and more likely to be congruent with the local traditions, cultural beliefs and values of their service users (Kpobi and Swartz, 2018; Li et al., 2020). Despite its ease of access and use, attempts to regulate the quality, safety and effectiveness of TH practice in developing countries have been difficult, as skills are often passed on through generations by word of mouth and often not documented (WHO, 2013). This may be attributed to beliefs that disclosing $\mathrm{TH}$ procedures may lead to a loss in their effectiveness as a treatment (Maluleka and Ngulube, 2018). Consequently, there seems to be a gradual disappearance of TH's use of herbs, which may be related to the modernisation of communities, deforestation and dominance of the biomedical model (Igoli et al., 2005), as well as the degrading effect of climate change, which has led to a significant loss of flora and fauna (Mbah and Fonchingong, 2019).

Labinjo et al. (2020) conducted a scoping review of 64 articles on perceptions, attitudes and cultural understandings of mental health in Nigeria. They found widespread beliefs about supernatural causes of MHCs such as divine punishment, evil spirits possession, witchcraft and sorcery as a significant cultural aspect for Nigerians. Of the 64 studies, 17 
identified TH and spiritual "homes" as the first treatment options. Within the Nigerian context, these "homes" are places of spiritual commitments and worship through continued prayers, fasting, prophecies and divine visions as therapeutic processes for healing MHCs (Labinjo et al., 2020). Consequently, traditional and religious healing institutions and their collaboration with biomedical services would help transform the Nigerian mental health sector and further address the global mental health treatment gap (Pham et al., 2020; Gureje et al., 2019; Jidong et al., 2020a, 2020b).

Little is known about why people with MHCs decide to use TH. However, the help-seeking behaviour of service users could be influenced by their literacy of MHCs. For instance, $\mathrm{Li}$ et al. (2020) found that highly educated women were less likely to use $\mathrm{TH}$ when compared to lesser educated women. The implication here is that the more educated a service-user is, the more likely are their values to be assimilated by Western ideas on biomedical orientation to MHCs and treatment. Although Li et al.'s (2020) study explored only women perspectives of $\mathrm{TH}$ that may not reflect the men or the general population, the study gave some insights about the potential influence of Western education and preferences for the use or non-use of TH.

Extending TH knowledge into the Nigerian mainstream public health care could be beneficial. For example, Kooreman and Baars (2012) study showed that general practitioners trained in alternative medicines showed service users had lower mortality rates, fewer hospital stays and fewer prescriptions of medications. Their study recommended adopting a hybrid approach that combines TH and Western biomedical model that could be more efficient and cost-effective. However, subjecting the Nigerian TH practice for scientific scrutiny and compatibility with the Western biomedical model may require methodological flexibility, as Hussain and Malik (2013) suggested, considering the beliefs around its spiritual aspects.

Perhaps, the methodological flexibility that Hussain and Malik (2013) refer to could be achieved by embracing an Afrocentric ideology, commonly referred to as Afrocentrism. According to Asante (2007), Afrocentrism is concerned about an African renaissance. This ideology attempts to redeem Africa's cultural values and traditions that were discredited as inferior by Eurocentric ideologies through slavery in the West or colonisation in Africa. When applied in the health field, Afrocentrism will thus be concerned about placing Africa's interests and values at the centre of interventions to achieve more effective and significant outcomes (Chawane, 2016; Jidong et al., 2020a, 2020b). In the Nigerian context, applying the Afrocentric ideology would require deconstructing the dominant biomedical discourse and embracing some cultural beliefs around $\mathrm{MHC}$ and $\mathrm{TH}$.

Informed by the Afrocentric ideology, the present study explores Nigerian mental health-care practitioners (MHCPs) and lay participants' (LPS) views regarding MHCs and $\mathrm{TH}$. Thus, the study's significance would enhance knowledge, thinking, and understanding concerning how MHCs are perceived among indigenous Nigerians with the broader aim of improving mental health and well-being. The rigour employed in this study's methodology strengthens its originality and innovative contribution to research and practice.

\section{Method/design}

\section{Research design}

This qualitative study adopted a critical realist and social constructionist lens to explore the views of MHCPs and LPS on MHCs and TH (Harper, 2011). Both the realist and constructionist features in the data sets were explored. The theoretical research lens acknowledges that the perception of social and cultural realities in the social realm widely depends on human beliefs and experiences (Burr, 2015). Therefore, the present study 
assumed that dominant narratives from the MHCPs and LPs may not be a direct representation of their realities but are in part influenced by knowledge of their realities as a product of shared history, language and space, which are essential in interpreting MHCs (Jidong et al., 2020a).

\section{Sampling/participants}

Purposive and snowball sampling techniques were used to recruit 53 participants $(\mathrm{MHCPS}=27$; LPS $=26)$. MHCPs are Western-trained professionals that include clinical psychologists $(n=13)$, psychiatrists $(n=7)$, psychiatric nurses $(n=2)$, guidance counsellors $(n=2)$, psychologist $(n=1)$ and a social worker $(n=1)$. MHCPs had a mean age of 12.1 years of working experiences in the psychiatric units of teaching hospitals, general hospitals and mental health counselling facilities in universities and environs. LPs were members of the public who did not have Indigenous or Western professional training in mental health care. However, LPs recruited had other types of experience, such as students $(n=16)$, public civil servants $(n=6)$, unemployed $(n=3)$ and self-employed entrepreneurs $(n=2)$.

Recruitment took place in four key cities of Nigeria, which reflects the country's rich and diverse ethnic people in Jos (Berom), Zaria (Hausa), Ado-Ekiti (Yoruba) and Enugu (Igbo) cities (Table 1). Participants were 32 men and 21 women with an age range of 18-65years. All participants self-identified as members of their given communities familiar with Indigenous native languages and cultural practices. In the ensuing section and following the qualitative research tradition of thick description (Ponterotto, 2006; Creswell and Miller, 2000), an attempt is made to unveil the process of participant selection and ethical consideration.

\section{Procedures for participants' recruitment and ethical considerations}

The research first obtained all the required ethical approvals from the University of East London (UEL) Research Ethics Committee, designated hospitals for interviewing mental health practitioners and psychology departments in each of the four Nigerian universities for approval of their research laboratories and office spaces for interviewing LPs. A six-step ethical procedure was followed during the participants' recruitment and interviews:

\section{Table 1 Sample interview questions}

Lay participants

a. What do you understand by a mental health condition?

Prompts: (i) How would you tell when someone is having an $\mathrm{MHC}$ ?

(ii) What would you say are the causes of MHCs within your ethnic community?

(iii) How do you think people from your community think about people with MHCs? b. Where do you think people from your community usually go for help/support/ treatment if they are experiencing MHCs? c. What would you say should be considered culturally important when helping someone from your community who needs psychological support?

d. What would be your opinion about the role of traditional healers and native doctors in mental health issues within your community or culture?
Mental health-care practitioners

a. What do you think are the common narratives about MHCs in this community?

Prompts: (i) What do you think are the dominant cultural beliefs about the causes of MHCs? (ii) Do these cultural/traditional beliefs affect their help-seeking behaviours for MHCs or your practice as a western-trained professional? b. Where do you think people from this community are most likely to go for help/ support/treatment if they are experiencing MHCs?

C. What would you say it should be considered culturally important when helping someone from this community who needs mental health support?

d. What is your opinion about the role of traditional healers or native doctors in mental health support for people in this community? 
Step 1: After local ethics were approved in Nigeria, an introductory letter was emailed to heads of services and psychology departments, requesting their permission to use their office spaces and laboratories to serve as data collection venues.

Step 2: After the venues for data collection were approved, printed research adverts were distributed to places frequented by "laypeople". These places included worship centres, restaurants and university campuses. In addition to the research adverts, participants' information sheets were physically distributed to intended volunteers who wanted to participate in the study. The participants that met the inclusion criteria were asked when it was convenient to participate in the study. The mental health practitioners were contacted in their places of work at university teaching hospitals. Subsequently, the date, time and venue for interviews were unanimously agreed with the lay and professional participants.

Step 3: At the beginning of the interview sessions, each participant was given a brief verbally, which further explained the nature of the study. Participants were also allowed to ask questions before interviews. Also, participants who wished to continue signed a hard copy of a consent form.

Step 4: A short questionnaire was administered to participants to collect demographic information such as age, gender, state of origin, level of education (and years of service for mental health practitioners), tribe or mother tongue, knowledge of native languages and the cultural practices of their ethnic groups.

Step 5: This stage involved the interview process, using the interview schedule (see Table 1 below). Interviews took place in quiet and confidential research laboratories and office spaces in the designated hospitals (for mental health practitioner interviewees) and the psychology departments in the Nigerian universities (for "lay" interviewees).

Step 6: Participants were given the opportunity, at the end of the interviews for debriefing to share their general thoughts regarding the interview process. The debriefing also clarified any concerns that participants raised after the interviews. Additional information about the research such as telephone helplines and contact details were given to participants, should they want to contact the researchers later after the interview.

\section{Methods of data collection and tools}

A semi-structured interview schedule was used for data collection. The interview schedule development was partly informed by reviewed literature and iteratively designed in line with the study's aims. Interview questions for MHCPs and LPs were slightly different to suit their varying perceptions and opinions.

The semi-structured interviews were designed to capture narrative data. Each interview lasting for approximately $55 \mathrm{~min}$ was audio-recorded and later transcribed verbatim. The transcripts were entered into an NVivo version 11-QSR data management programme to assist with data organisation, including coding and categorisation.

\section{Data analysis}

Thematic analysis was adopted executing the six-stage guidelines recommended by Braun and Clarke $(2013,2006)$. This included familiarisation, coding, generating themes, reviewing themes, defining and naming themes and writing up. Also, coding and themes' development were iteratively conducted using both inductive and deductive analysis of the data transcripts (Fereday and Muir-Cochrane, 2006).

Both inductive and deductive findings resonated in the data analysis. For instance, inductive data analysis was primarily data-driven based on participants' defined meanings in the data transcripts rather than tailoring to existing theories and concepts 
(Smith, 2015). This was particularly beneficial in identifying emergent themes that have implicit content (Bryman, 2012). The reviewed literature potentially influenced deductive findings. Identified vivid expressions were used as supporting extracts for the emerging themes in the results section. The study's epistemology and exploratory scope are compatible with the semi-structured interviews and thematic analysis adopted (Burr, 2015; Willig, 2013; Harper, 2011).

\section{Ethical approvals}

Ethical approval for the study was received from a university in the UK and four university teaching hospitals and institutions in the designated cities of data collection in Nigeria.

\section{Results/themes}

Participants were 32 men and 21 women with an age range of 18-65 years (Table 2).

Three themes emerged from the interviews:

1. existing cultural beliefs about MHCs as spiritual curse;

2. description of TH as the first treatment modality for MHCs; and

3. perceived stigma associated with MHCs and help-seeking behaviours.

See Figure 1 below.

\section{Table 2 Research participants recruited to the study}

\begin{tabular}{lccr} 
Cities (ethnicity) & Laypeople (LP) & Mental health-care practitioners (MHCP) & Total \\
\hline Ado-Ekiti (Yoruba) & $n=7$ & $n=7$ & 14 \\
Enugu (Igbo) & $n=6$ & $n=7$ & 13 \\
Jos (Berom) & $n=7$ & $n=6$ & 13 \\
Zaria (Hausa) & $n=7$ & $n=6$ & 13 \\
Total & 27 & 26 & 53
\end{tabular}

\section{Figure 1 Showing themes that emerged from the analysed data}

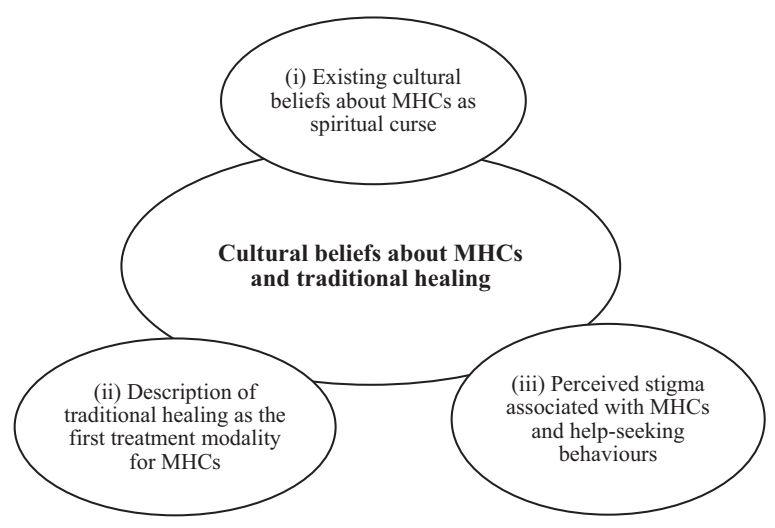




\section{(i) Existing cultural beliefs about MHCs as spiritual curse}

Existing studies have suggested a perceived intrinsic link between MHCs and a spiritual curse in African communities (Igberase and Okogbenin, 2017; Rao, 2009; Stompe et al., 2006; Okpalauwaekwe et al., 2017; Jegede, 2005). This was a belief shared between MHCPs and LPs in this study. An MHCP said:

[Persons suffering from MHCs] are individuals that have committed a serious crime or rather they have offended the gods, their gods, and the gods are now chasing them, or they have done something wrong to one individual, and the person decided to punish them [with MHCS] [MHCP, 1].

Similarly, an LP explained: When you see someone who is mentally derailed to an extent whenever the person is speaking; from being dirty to walking naked in the streets- the first assumption of people here is that the person is inflicted [...] by fellow humans, this person has been inflicted with madness [LP, 1].

First, it appears that the cultural definition of someone suffering from an $\mathrm{MHC}$ is attributed to the individual's inability to speak coherently or unable to look after themselves. Second, the "gods" and "aggrieved individuals" are construed as playing a central role in the infliction of MHCs. The above extracts suggest how external factors independent of the individual's control is perceived as integral. That is, MHCs is externally cursed rather than attributable to a person's characteristics. An interviewed MHCP narrated the perception of service users' beliefs about MHCs in the following extracts:

When we started therapy, the patient was so worried, but the first thing that came to my mind was that he was a high profile politician, well-educated, the first thing that came to the politician's mind was that his situation was a spiritual attack and of course, he was sacrificing goats, he was sacrificing cows, performing all sorts of rituals to walk away the evil [MHCP, 2].

It is apparent in the above extract that these beliefs are likely to be inherent among people of different socio-economic, political and educational backgrounds. As such, Westerntrained practitioners report finding innovative ways of doing culturally sensitive therapy that acknowledges and accommodates these beliefs alongside their mainstream practice. Furthermore, the belief about spiritual curses of MHCs appeared to be genuinely developed by the Western-trained MHCP who have worked for several years in the communities. An interviewed clinical psychologist said:

Personally now, over the years there was a time I discounted that aspect [spiritual curse of MHCs], but as I keep interacting with people closely at that community-level, I understand we need to adopt a holistic approach to issues [of mental health] [MHCP, 4].

The above extract showed a re-emergent of an MHCP's agreement with the belief about MHCs as a spiritual curse after several years of working experience in the shared social space with service users in the locality. These beliefs from the practitioner's perspective might not be congruent with the Western clinical training and practice. However, a possible explanation for this view might be associated with practices that allow for a holistic approach by potentially accommodating both Western biomedical model and the spiritual interventions such as the $\mathrm{TH}$ for $\mathrm{MHCs}$.

A salient perspective on the manifestation of beliefs about the spiritual curse of MHCs by an interviewed MHCP who said:

Somebody who stole money and was earlier informed that if you steal this money, you will have a problem with poor senses which is going to be inflicted by the gods [.. .] when an individual has a background history of emotional issues in a family either psychosis or schizophrenia which has been a link to the genetic chromosomes in the body system. Now, over stressful events or stealing the money, coming down with guilty feelings, and those traces and the programmed genetic traces are already in the background would have triggered [...] mental illness, [and] 
then, the person will now think it is the gods that have inflicted those problems on him or her [MHCP, 6].

The above extract exemplified views relating to the "law of karma" (Reichenbach, 1988), which may suggest that one's wrongdoing may have the potential to trigger MHCs.

\title{
(ii) Description of traditional healing as the first treatment modality for MHCs
}

Western biomedical models seem to dominate the research landscape and training for mental health treatment. However, its application within the Nigerian cultural context seems to be limited. A common pattern in the data set was the perceived view that TH serves as the first point of call for mental health help-seeking behaviours. Data analysis showed that traditional healers were important figures in local communities and widely consulted by potential service users. Two participants said:

\begin{abstract}
Most people do not see the clinician as the first point of call when they have symptoms of mental illnesses, they, first of all, go to these healers and when the symptoms have become worse that is when they come to the clinicians [MHCP, 1].

We cannot discountenance or deny the fact that this people [THS] are closer to the society more than the western-trained counterparts [MHCP] in terms of mental healthcare provision as the case may be-now because they are closer to the society that means you cannot disregard them. Because they first consult them [THs] before the western counterpart. Their first point of contact will have been this set of people [THs] that means their mental health perspective begins there, about their beliefs, about their treatment responds or treatment reaction stem from the understanding that these people heal them [MHCP, 5].
\end{abstract}

The reality captured here is that the traditional healers are closer to the people and are readily available in their communities. This results in $\mathrm{TH}$ becoming the primary source of care for potential service users with Western care as secondary. A possible explanation for such common beliefs and help-seeking behaviours relates to how Western treatment for $\mathrm{MHCs}$ is construed as limited in serving culture-specific purposes. This was more delineated in the following extracts where some participants said:

You know it is not every sickness that can be cured by English medicine. So, there are good herbs that our forefathers used and those traditional herbalists they got the idea from their forefathers too, and they have been utilising it to help people out" [LP, 2]. "There are some psychological problems- that cannot be treated psychologically [by orthodox mental healthcare system], they are meant to be treated the ((cultural)) way [TH] because they are mysterious, you don't expect someone with a spiritual problem to go for psychotherapy, they are definitely wasting their time, so there are some MHCs that cannot be treated psychologically [LP, 3].

Some MHCs are believed as only treatable with Indigenous herbs and $\mathrm{TH}$ process. $\mathrm{TH}$ is construed as organically inherent in the people and historically transmitted from one generation to another since time immemorial. Some MHCs are construed as mysterious and, therefore, believed that Western care for treating such illnesses would be unsuccessful. More on the indigenous herbal treatment for MHCs, some of the participants said:

[Herbs] could cure madness, there are even herbs that are made for depression- depression can lead to hypertension, so there are herbs they made to prevent that" [LP, 3]. "There are some certain herbs they used, there is something they called ((speaking native)) it actually contains some antipsychotics with sedative effects, they actually boil it, they know the patient that has it. I don't know how they discover that patient that has psychosis, and they are actually violent, so once they take it and they sniff the effusion from the boiled leaves you give the patient to drink and to bath with it after which the patient actually calms down [MHCP, 5].

Indigenous herbs are believed to have medical values similar to antidepressant and antipsychotics to treat MHCs such as depression and psychosis. Although, the process of 
diagnoses and identification of specific MHCs is not explicit, sniffing of boiled medicinal leaves is portrayed as the physical process of herbal treatment. The affordability and costeffectiveness of TH were also acknowledged in the data.

[We often use TH] because is too cheap for us, if we go to the herbalists they would just request some few things that they would bring, and they would try to solve it, [THs] do some incantation and some sacrifice, and it will help [in healing MHC] [LP, 4].

In this extract, TH is construed as useful, helpful and affordable. The spiritual aspects of TH entail incantations in strange words using sacrificial farm produce such as yam, cocoyam, cassava, palm wine or livestock such as chicken, goat or ramp. TH's cost is believed to be more affordable and cheaper compared to Western biomedical care for MHCs.

\section{(iii) Perceived stigma associated with MHCs and help-seeking behaviours}

Current findings showed reoccurring reports of the high levels of stigma associated with MHCs. Two LPs said:

In [our] tradition we actually refer to it [MHCs] as a taboo, in fact, it comes with a social stigma, it comes with a social stigma that one tends to, or attribute it to something that runs in hereditary form, [for instance, if] you want to get married in that house [family], they want to check whether that family has a history of psychotic disorder, has a history of what we may call madness, or whether madness runs in their blood [LP, 5].

[when] you are not able to reason well or mentally, just to talk about madness, you know, you have become scary, the things you do all the time create fear, people would not want to come close to you [...] You would become like an outsider everybody would be running away from you $[L P, 6]$.

The persistent stigma and lack of community support for service users even after decades of full recovery from MHCs may suggest a lack of awareness about MHCs. Speaking on the stigma associated with help-seeking behaviours, another interviewee said:

Nobody wants to be seen coming to the psychiatry facility. It is so bad that people find it difficult to open a pure psychiatric private practice [...] You will never see a patient coming to that facility unless if you name it just like any other hospital [MHCP, 2].

Because of the high levels of stigma for people with MHCs, as shown in the above extract, most potential service users would not want to have any form of association with mental health facilities. It is apparent that Western mental health services are highly stigmatised and discriminated upon although data analysis did not show if similar attitudes are extended to the TH facilities. However, any form of mental health service provision in communities may require all forms of confidentiality.

\section{Discussion}

The present study explored Nigerian MHCPs and LPs' views regarding MHCs and TH.

First, the existing cultural beliefs about MHCs as spiritual curse were revealed in both LPs and MHCPs alike across the entire data sets. The spiritual curse is reportedly associated with forces beyond the individual, such as ancestors or magic. The ancestors and other powerful spirits could inflict MHCs on criminals or other persons who commit taboos in the community. This is consistent with the findings of Kabir et al. (2004) and Okpalauwaekwe et al. (2017). Kabir et al. (2004) conducted a study in Karfi village of Northern Nigeria with a sample size of 250 participants. The survey showed major causes of MHCs with $19 \%$ who believed it is divine wrath or God's will, and $18 \%$ who believed it as spirit or magic possession. 
It is believed that an enemy could hire "false" ritualists to inflict MHCs on another person because of a dispute. The beliefs seemed to be common regardless of the political, socioeconomic or educational backgrounds. This is slightly contrary to the findings in the study of Igberase and Okogbenin (2017) who surveyed informal caregivers and relations of schizophrenic service users in Midwest Nigeria, which showed $72 \%$ of 200 uneducated participants endorsed spiritual curse of MHCs. Although many factors might have accounted for the research outcomes in the two studies such as the quantitative features in the study of Igberase and Okogbenin (2017) and perhaps, the qualitative scope of the present study. However, what is common in the two studies is the belief in the spiritual curse of MHCs. Although these beliefs are likely to be debunked by Western biomedical clinicians, the present study showed some Western-trained MHCPs revealed that their several years of working with Indigenous service users have changed their perspectives. These practitioners expressed a genuine belief in the contribution of the spiritual curse of MHCs.

An alternative explanation to the popular beliefs on the spiritual curses of MHCs could be derived from the "law of karma" (Reichenbach, 1988), which suggest that mere involvement in cultural taboos may be a natural trigger to mentally venerable individuals. Thus, uncontrollable negative automatic thoughts of a guilty mind because of cultural taboo involvement could activate predisposing factors and mysterious beliefs about MHCs. This line of argument was made in the study of Rao (2009) and Stompe et al. (2006) that wrongdoings may trigger MHCs similar to the "law of karma", that is, anything an individual does may create directly proportional energy capable of affecting the person in some forms (Reichenbach, 1988).

Second, findings showed a description of $\mathrm{TH}$ as the first point of contact for seeking mental health treatment, and service users may only seek Western care as a last resort when $\mathrm{TH}$ was not experienced as helpful. A similar finding was noted in the study of Takim et al. (2013) who reported that TH is the favourite and the first point of contact for many Nigerians seeking mental health care. Furthermore, Kabir et al. (2004) showed that 34\% of participants were more inclined towards spiritual healing through exorcism and herbal medicine. Essentially, the present findings showed TH system with the use of Indigenous herbs is beneficial and perceived as helpful for treating MHCs for several generations, which is equally similar to the research outcomes in the study of Sarfo (2015).

The compelling usage of TH systems might have been a consequence of the cultural beliefs about spiritual curses of MHCs and the subsequent use of $\mathrm{TH}$, which is also believed to be spiritually inclined. The traditional healers intercede or negotiate mental wellness of their service users with the spirits that have powers to heal. This was similarly found in the study of Kpobi and Swartz (2018), which suggests that MHCs were diagnosed based on the act of enquiring information from the ancestors. The ancestral spirits describe processes of engaging in rituals to communicate with spiritual beings. Although Kpobi and Swartz's (2018) study was conducted in Ghana, both Ghana and Nigeria appeared to share similar cultures and traditions. Aside from the notion that Western biomedical care in Nigeria is very limited and scarce in contrast to the TH that appeared to be readily available and accessible in communities and their linguistic and cultural compatibility (Kpobi and Swartz, 2018; WHO, 2013). These features of TH might have accounted for the high patronage by service users. More so, the recent study by Li et al. (2020) revealed an $81.6 \%$ usage of $\mathrm{TH}$ among adult women in Nigeria. The study focussed on women that may not reflect the actual general practice of health-care-seeking behaviours of Nigerian general public. Nevertheless, the high usage of $\mathrm{TH}$ is enormous.

Third, this study showed a perceived stigma associated with MHCs and help-seeking behaviours against potential service users. The current cultural practices do not seem to encourage open conversation on mental health issues. For example, people with some history of MHCs might be denied access to some rights such as marriage or significant 
public roles despite several years of recovery. The consequence of which is that some families try to conceal mental ill cases of family members to avoid such stigma and discrimination. This finding was previously supported in the study of Lasebikan (2016) who explored how people's cultural beliefs influence their interpretation of MHCs, associate stigma and help-seeking behaviours. This high level of stigma may explain the current lack of openness on mental health issues, and thus, associated with the current low levels of mental health awareness in the Nigerian communities, especially in the rural areas. Similarly, both Abdulmalik et al. (2016) and Gureje et al. (2015) opined that lack of available mental health information is a considerable challenge and recommended awareness creation as an essential step in bringing mental health information closer to the people in their localities.

\section{Limitations of this study}

One of the key findings in the present study was the usefulness of TH system in Nigeria. However, traditional healers were not interviewed in this study, thereby creating a fundamental limitation. Another limitation is that this study did not explore the actual experiences of service users receiving TH services. Although at the time of the study, LPs were not receiving $\mathrm{TH}$; however, they were considered as potential service users either based on previous personal experience, their awareness of close relations or community members who had received or experienced $\mathrm{TH}$.

This study's strength includes richer and deeper qualitative data collected using semistructured interviews that allowed participants' defined meanings that emerged in the data sets. Another strength is participants' recruitment in strategic locations that accommodated the country's diverse Indigenous cultural perspectives, including the three majority ethnic groups of Hausa, Yoruba and Igbo and the minority Berom ethnic people. More so, the study's novelty could be attributed to the inclusion of both MHCPs and LPS, which provided robust and multifaceted data sets. Finally, based on the authors' knowledge, this is the first study that has explored the perspectives of LPs and MHCPs on MHCs and TH.

\section{Recommendations}

Future studies could further explore these experiences and perceptions with the involvement of TH systems in Nigeria. This is important because it is culturally, historically and linguistically compatible with the Indigenous people. Most fundamentally, TH is highly patronised by the community members, yet there seems to be not much public investment in the mental health sector. It will be beneficial to assign appropriate budgetary allocation for both treatment and research to revamp the TH sector. To minimise unhelpful and inhuman TH practices, a realistic mechanism could be established to scrutinise, register and monitor the genuineness and effectiveness of traditional healers in the country. National reorientation platforms could establish an open conversation on mental health issues as a preliminary step for creating awareness and combating stigma and discrimination of service users.

\section{Implications for professionals working in mental health}

The implication of this paper for clinical practice in the foreseeable future is for service providers in Nigeria to embrace a culture-sensitive practice that incorporates Indigenous cultural beliefs and TH for MHCs. For example, the Western biomedical mental health practice in Nigeria could usefully harness cultural voices to shape their definition, diagnosis and treatment of MHCs using culturally appropriate remedies or interventions.

This study's contributions are twofold. First, this paper illustrates a potential trajectory between cultural beliefs about MHC as a spiritual curse and the prioritisation of $\mathrm{TH}$. TH for MHCs is believed to be rooted in natural and spiritual forces. Second, this study showed 
that culture-specific stigma and discrimination associated with MHCs could limit service users' traditional rights and other public benefits.

\section{Conclusions}

Cultural beliefs about the curse of MHCs influence how LP and MHCPs perceive TH's contribution to the Nigerian mental health-care system. TH services have been in use across generations and are believed to be helpful and culturally compatible. Innovative concepts could be adapted such as a hybrid approach that harmonises the useful principles and concepts of the Western biomedical and Nigerian TH practices to increase access to mental health care and further address the increasing local care needs of the Indigenous population. The implications of diversifying mental health-care practice to reflect the Indigenous laypeople's beliefs may mean an increase in its accessibility and acceptability. Nigerian cultural beliefs and TH should be better understood to work towards a collaborative approach in treating and managing the increasing cases of MHCs. The likely dismissal of $\mathrm{TH}$ in the mainstream biomedical services may neglect the long history and evidential benefits of TH. Nigerian healthcare services could use evidence-based approaches to draw Indigenous knowledge, cultural beliefs and TH to build a healthier and sustainable mental health-care system.

\section{References}

Abdulmalik, J., et al. (2019), "Sustainable financing mechanisms for strengthening mental health systems in Nigeria", International Journal of Mental Health Systems, Vol. 13 No. 1, pp. 13-38.

Abdulmalik, J., Kola, L. and Gureje, O. (2016), "Mental health system governance in Nigeria: challenges, opportunities and strategies for improvement", Global Mental Health, Vol. 3 No. 9, pp. 1-11.

Anyebe, E.E., et al., (2019), "Current status of mental health services at the primary healthcare level in Northern Nigeria", Administration and Policy in Mental Health and Mental Health Services Research, Vol. 46 No. 5, pp. 620-628.

Asante, M.K. (2007), An Afrocentric Manifesto: Toward an African Renaissance, Cambridge: Polity Press.

Borokini, T.I. and Lawal, I.O. (2014), "Traditional medicine practices among the Yoruba people of Nigeria: a historical perspective", Journal of Medicinal Plants Studies, Vol. 2 No. 6, pp. 20-33.

Braun, V. and Clarke, V. (2006), "Using thematic analysis in psychology", Qualitative Research in Psychology, Vol. 3 No. 2, pp. 77-101.

Braun, V. and Clarke, V. (2013), Successful Qualitative Research: A Practical Guide for Beginners, Los Angeles: Sage.

Bryman, A. (2012), Social Research Methods, New York, NY: Oxford University Press Inc.

Burr, V. (2015), Social Constructionism, 3rd ed. London: Routledge.

Charlson, F.J., Diminic, S., Lund, C., Degenhardt, L. and Whiteford, H.A. (2014), "Mental and substance use disorders in Sub-Saharan Africa: predictions of epidemiological changes and mental health workforce requirements for the next 40 years", Public Library of Science, Vol. 9 No. 10, p. e110208.

Chawane, M. (2016), "The development of Afrocentricity: a historical survey", Yesterday and Today, No. 16, pp. 78-99.

Creswell, J.W. and Miller, D.L. (2000), "Determining validity in qualitative inquiry", Theory into Practice, Vol. 39 No. 3, pp. 124-130.

Fereday, J. and Muir-Cochrane, E. (2006), "Demonstrating rigor using thematic analysis: a hybrid approach of inductive and deductive coding and theme development", International Journal of Qualitative Methods, Vol. 5 No. 1, pp. 80-92.

Gureje, O., Lasebikan, V.O., Kola, L. and Makanjuola, V.A. (2006), "Lifetime and 12-month prevalence of mental disorders in the Nigerian survey of mental health and Well-Being", The British Journal of Psychiatry, Vol. 188 No. 5, pp. 465-471. 
Gureje, O., Abdulmalik, J., Kola, L., Musa, E., Yasamy, M.T. and Adebayo, K. (2015), "Integrating mental health into primary care in Nigeria: report of a demonstration project using the mental health gap action programme intervention guide", BMC Health Services Research, Vol. 15 No. 1, pp. 1-8.

Gureje, O., Seedat, S., Kola, L., Appiah-Poku, J., Othieno, C., Harris, B., Makanjuola, V., Price, L.N., Ayinde, O.O. and Esan, O. (2019), "Partnership for mental health development in Sub-Saharan Africa (PaM-D): a collaborative initiative for research and capacity building", Epidemiology and Psychiatric Sciences, Vol. 28 No. 4, pp. 389-396.

Harper, D. (2011), Choosing a Qualitative Research Method. In, "Choosing a qualitative research method. Qualitative research methods in mental health and psychotherapy: a guide for students and practitioners", Sussex: John Wiley \& Sons, pp. 83-97.

Horwitz, A.V. (2020), Creating Mental IIIness, University of Chicago Press.

Hussain, S. and Malik, F. (2013), "Integration of complementary and traditional medicines in public health care systems: challenges and methodology", Journal of Medicinal Plants Research, Vol. 7 No. 40, pp. 2952-2959.

Igberase, O. and Okogbenin, E. (2017), "Beliefs about the cause of schizophrenia among caregivers in Midwestern Nigeria", Mental IIIness, Vol. 9 No. 1, pp. 22-27.

Igoli, J.O., et al., (2005), "Traditional medicine practice amongst the Igede people of Nigeria. Part II", African Journal of Traditional, Complementary and Alternative Medicines, Vol. 2 No. 2, pp. 134-152.

Jegede, A. (2005), "The notion of 'were' in Yoruba conception of mental illness", Nordic Journal of African Studies, Vol. 14 No. 1, pp. 117-126.

Jidong, D.E. and Sanger, R. (2018), "Exploring critical understanding of hoarding distress among elderly people in Nigeria: a review of Smail's impress of power model", International Journal of Health Sciences, Vol. 6 No. 2, pp. 46-51.

Jidong, D.E., Tribe, R. and Gannon, K.N. (2020a), "Challenges faced by mental health interpreters in east London: an interpretative phenomenological analysis", Counselling Psychology Review, Vol. 35 No. 2, pp. 40-51.

Jidong, D.E., Husain, N., Francis, C., Murshed, M., Roche, A., Ike, T., Karick, H., Dagona, Z.K., Pwajok, J. Y., Nyam, P.P., Mwankon, S.B. and Gumber, A. (2020b), "Mental health experiences of mothers in Jos Nigeria: an interpretative phenomenological analysis", SAGE Open Medicine, Vol. 8, pp. 1-8., doi: $10.1177 / 2050312120970714$.

Kabir, M., lliyasu, Z., Abubakar, I.S. and Aliyu, M.H. (2004), "Perception and beliefs about mental illness among adults in Karfi village, Northern Nigeria", BMC International Health and Human Rights, Vol. 4 No. 1, pp. 1-5.

Kooreman, P. and Baars, E.W. (2012), "Patients whose GP knows complementary medicine tend to have lower costs and live longer", The European Journal of Health Economics, Vol. 13 No. 6 , pp. 769-776.

Kpobi, L. and Swartz, L. (2018), "That is how the real mad people behave': Beliefs about and treatment of mental disorders by traditional medicine-men in Accra", Ghana. International Journal of Social Psychiatry, Vol. 64 No. 4, pp. 309-316.

Labinjo, T., Serrant, L., Ashmore, R. and Turner, J. (2020), "Perceptions, attitudes and cultural understandings of mental health in Nigeria: a scoping review of published literature", Mental Health, Religion \& Culture, Vol. 23 No. 7 , pp. 606-624.

Lasebikan, V.O. (2016), "Cultural aspects of mental health and mental health service delivery with a focus on Nigeria within a global community", Mental Health, Religion \& Culture, Vol. 19 No. 4, pp. 323-338.

Li, S., et al., (2020), "Traditional medicine usage among adult women in Ibadan, Nigeria: a crosssectional study", BMC Complementary Medicine and Therapies, Vol. 20 No. 1, pp. 1-7.

Maluleka, J.R. and Ngulube, P. (2018), "The preservation of knowledge of traditional healing in the Limpopo province of South Africa”, Information Development, Vol. 34 No. 5, pp. 515-525.

Mbah, M. and Fonchingong, C. (2019), "Curating indigenous knowledge and practices for sustainable development: possibilities for a socio-ecologically-minded university", Sustainability, Vol. 11 No. 15, p. 4244. 
Okpalauwaekwe, U., Mela, M. and Oji, C. (2017), "Knowledge of and attitude to mental illnesses in Nigeria: a scoping review", Integrative Journal of Global Health, Vol. 1 No. 5, pp. 1-14.

Onyemelukwe, C. (2016), "Stigma and mental health in Nigeria: some suggestions for law reform", Journal of Law, Policy \& Globalisation, Vol. 55, pp. 63-68.

Oshodi, Y.O., Abdulmalik, J., Ola, B., James, B.O., Bonetto, C., Cristofalo, D., Van Bortel, T., et al. (2014), "Pattern of Experienced and Anticipated Discrimination among People with Depression in Nigeria: a Cross-Sectional Study", Springer, Vol. 49 No. 2, pp. 259-266.

Pham, T.V., Koirala, R., Wainberg, M.L. and Kohrt, B.A. (2020), "Reassessing the mental health treatment gap: what happens if We include the impact of traditional healing on mental illness?", Community Mental Health Journal, pp. 1-15.

Ponterotto, J.G. (2006), "Brief note on the origins, evolution, and meaning of the qualitative research concept thick description", The Qualitative Report, Vol. 11 No. 3, pp. 538-549.

Rao, A.V. (2009), "Depressive illness and guilt in Indian culture", Asian Journal of Psychiatry, Vol. 2 No. 2, pp. 84-86.

Reichenbach, B.R. (1988), "The law of karma and the principle of causation", Philosophy East and West, Vol. 38 No. 4, pp. 399-410.

Sankoh, O., Sevale, S. and Weston, M. (2018), "Mental health in Africa", The Lancet Global Health, Vol. 6 No. 9, pp. e954-e955.

Sarfo, I.A. (2015), "The power of beliefs on health seeking behaviour: implication for therapeutic relationships for cardiovascular care", European Journal of Medicine, Vol. 10 No. 4, pp. 195-207.

Smith, J.A. (2015), Qualitative Psychology: A Practical Guide to Research Methods, London: Sage

Stompe, T.S., et al. (2006), "Delusions of guilt: the attitude of Christian and Muslim schizophrenic patients toward good and evil and the responsibility of men", Journal of Muslim Mental Health, Vol. 1 No. 1 , pp. 43-56.

Suleiman, D. (2016), "Mental health disorders in Nigeria: a highly neglected disease", Annals of Nigerian Medicine, Vol. 10 No. 2, pp. 47-48.

Takim, A.O., David, G.I. and Pefun, J.N. (2013), "African cultural practices and health implications for Nigeria rural development", International Review of Management and Business Research, Vol. 2 No. 1, pp. 176-183.

Weye, N., Christensen, M.K., Momen, N.C., Iburg, K.M., Plana-Ripoll, O. and McGrath, J.J. (2020), "The global burden of disease methodology has been good for mental disorders: but not good enough”, The Canadian Journal of Psychiatry, Vol. 65 No. 2, pp. 102-103.

Willig, C. (2013), Introducing Qualitative Research in Psychology, England: McGraw-Hill Education.

World Health Organisation (2001), "The world health report 2001: mental health, new understanding, new hope [online]", available at: www.who.int/whr/2001/en/

World Health Organisation (2013), "WHO traditional medicine strategy 2014-2023".

World Health Organization (2019a), "Making the investment case for mental health: a WHO (no. WHO/ UHC/CD-NCD/19.97)".

World Health Organization (2019b), "The WHO special initiative for mental health ( 2019-2023): universal health coverage for mental health (no. WHO/MSD/19.1). world health organization", available at: https://apps.who.int/iris/bitstream/handle/10665/310981/WHO-MSD-19.1-eng.pdf? sequence $=1$ \&is Allowed $=\mathrm{y}$

Worldometers (2020), "Nigeria population [online]", available at: www.worldometers.info/worldpopulation/nigeria-population/ (accessed 13 December 2020).

\section{Further reading}

Phillimore, J., et al., (2018), "Bricolage as conceptual tool for understanding access to healthcare in superdiverse populations”, Social Theory Health, Vol. 17 No. 2, pp. 231-252. 
Author affiliations

Dung Ezekiel Jidong is based at the Department of Psychology, Nottingham Trent University, Nottingham, UK.

Di Bailey is based at the Research and Strategic Partnerships, Nottingham Trent University, Nottingham, UK.

Tholene Sodi is based at the Department of Psychology, University of Limpopo, Polokwane, South Africa.

Linda Gibson is based at the Institute of Health and Allied Professions, Nottingham Trent University, Nottingham, UK.

Natéwindé Sawadogo is based at the University Institute for Initiale and Continuing Training, Thomas Sankara University, Gonsin, Burkina Faso.

Deborah Ikhile is based at Brighton and Sussex Medical School, University of Sussex, Brighton, UK.

David Musoke is based at the Department of Disease Control and Environmental Health, Makerere University, Kampala, Uganda.

Munyaradzi Madhombiro is based at the Department of Psychiatry, University of Zimbabwe, Harare, Zimbabwe.

Marcellus Mbah is based at the Nottingham Institute of Education, Nottingham Trent University, Nottingham, UK.

\section{Corresponding author}

Dung Ezekiel Jidong can be contacted at: dung.jidong@ntu.ac.uk

For instructions on how to order reprints of this article, please visit our website: www.emeraldgrouppublishing.com/licensing/reprints.htm

Or contact us for further details: permissions@emeraldinsight.com 